\title{
Construction and characterization of a recombinant yellow fever virus stably expressing Gaussia luciferase
}

\author{
TELISSA C. KASSAR ${ }^{1, *}$, TEREZA MAGALHÃES ${ }^{1}$, JOSÉ V.J.S. JÚNIOR ${ }^{1}$, AMANDA G.O. CARVALHO ${ }^{1}$, \\ ANDRÉA N.M.R. DA SILVA ${ }^{1, * *}$, SABRINA R.A. QUEIROZ ${ }^{1, * * *}$, GIOVANI R. BERTANI ${ }^{2}$ and LAURA H.V.G. GIL ${ }^{1}$ \\ ${ }^{1}$ Departamento de Virologia e Terapia Experimental, Centro de Pesquisas Aggeu Magalhães/CPqAM, Fundação \\ Oswaldo Cruz/FIOCRUZ, Av. Professor Moraes Rego, s/n, Cidade Universitária, 50740-465 Recife, PE, Brazil \\ ${ }^{2}$ Departamento de Bioquímica, Universidade Federal de Pernambuco/UFPE, Av. Professor \\ Moraes Rego, s/n, Cidade Universitária, 50670-420 Recife, PE, Brazil
}

Manuscript received on April 15, 2016; accepted for publication on June 20, 2016.

\begin{abstract}
Yellow fever is an arthropod-borne viral disease that still poses high public health concerns, despite the availability of an effective vaccine. The development of recombinant viruses is of utmost importance for several types of studies, such as those aimed to dissect virus-host interactions and to search for novel antiviral strategies. Moreover, recombinant viruses expressing reporter genes may greatly facilitate these studies. Here, we report the construction of a recombinant yellow fever virus (YFV) expressing Gaussia luciferase (GLuc) (YFV-GLuc). We show, through RT-PCR, sequencing and measurement of GLuc activity, that stability of the heterologous gene was maintained after six passages. Furthermore, a direct association between GLuc expression and viral replication was observed $\left(\mathrm{r}^{2}=0.9967\right)$, indicating that measurement of GLuc activity may be used to assess viral replication in different applications. In addition, we evaluated the use of the recombinant virus in an antiviral assay with recombinant human alfa- $2 \mathrm{~b}$ interferon. A $60 \%$ inhibition of GLuc expression was observed in cells infected with YFV-GLuc and incubated with IFN alfa-2b. Previously tested on YFV inhibition by plaque assays indicated a similar fold-decrease in viral replication. These results are valuable as they show the stability of YFV-GLuc and one of several possible applications of this construct.
\end{abstract}

Key words: Gaussia luciferase, homologous recombination in yeast, reporter gene, yellow fever virus.

Correspondence to: Laura Helena Vega Gonzales Gil

E-mail: laura@cpqam.fiocruz.br

Present address: *Departamento de Medicina Veterinária Preventiva, Escola de Veterinária, Universidade Federal de Minas Gerais, Avenida Antônio Carlos, 6627, 31270-901 Belo Horizonte, MG, Brazil

**Laboratório de Virologia, Instituto de Ciências

Biológicas, Universidade Federal do Pará, Rua Augusto

Corrêa, 01, Guamá, 66075-110 Belém, PA, Brazil

${ }^{* * *}$ Departamento de Medicina Veterinária, Faculdade de Zootecnia e

Engenharia de Alimentos, Universidade de São Paulo, Avenida Duque Caxias Norte, 225, Jardim Elite, 13635-000 Pirassununga, SP, Brazil

\section{INTRODUCTION}

Yellow fever is an infectious disease caused by the yellow fever virus (YFV), a flavivirus (Family Flaviviridae), transmitted to humans by biting infected female mosquitoes of the genera Aedes, Sabethes and Haemagogus (Hanley et al. 2013). YFV is endemic in countries of Africa and South America and is maintained mainly through sylvatic 
transmission cycles among nonhuman primates and sylvatic mosquitoes. However, several, often disastrous, urban transmission cycles involving the mosquito Aedes aegypti as the main vector have occurred and the risk of urban outbreaks remains a threat, especially in regions where viruses and vectors are found (Hanley et al. 2013).

Yellow fever in humans can be divided into three clinical stages: infection, remission and intoxication (Ishikawa et al. 2014). After an incubation period of 3-6 days symptoms like headache, fever and myalgia may be observed, which corresponds to the viremic period. After the infection period, some patients may develop the remission period, presenting liver and renal failure. An intoxication period may follow, characterized by hemorrhagic fever and organ failure. Approximately 15\% of cases develop moderate/severe manifestations. The World Health Organization estimates that 200,000 cases and 30,000 deaths occur annually, mostly in Africa (Ishikawa et al. 2014).

Despite the availability of a human vaccine against YFV, the virus continues to pose a threat to human populations, reaching mortality rates of $20-50 \%$ in urban cycles. The currently available vaccine is based on the live-attenuated strain 17D or the substrains 17DD and 17D-204, and although it reaches high protection levels in vaccinees, it may cause rare, severe side effects leading to viscerotropic disease, especially in risk groups (Monath et al. 2015). The other control methods for arthropod-borne viral diseases such as yellow fever are: 1) anti-vector strategies, which have been proven highly difficult in the case of extremely adapted species such as Ae. aegypti; and 2) antiviral drugs. For the latter, the antiviral medications available are ineffective against YFV (Monath et al. 2015) and the search for novel effective medications is an active field of research.

The YFV is an enveloped, single-stranded, positive-sense RNA virus, with a genome of approximately $11 \mathrm{~Kb}$, capped at its 5' end and non- polyadenylated at its 3 ' end. It encodes, within one open-reading frame, three structural proteins at the $\mathrm{N}$-terminal portion (capsid-C, pre-membrane-prM, envelope-E) and seven non-structural proteins at the C-terminal portion (NS1, NS2a, NS2b, NS3, NS4a, NS4b, and NS5) of the polyprotein. Additionally, the YFV genome has functional non-coding terminal ends (5' and 3' untranslated regions-UTRs) that are involved in several viral features such as genome replication and protein translation (Lindenbach et al. 2013).

In the 1950s, it was discovered that the nucleic acid of some animal viruses was infectious. Later on, experiments showed that transfection of live, susceptible cells or hosts with the genome of several positive RNA viruses led to the production of infective viruses (Aubry et al. 2015). These discoveries have allowed the development of powerful viral molecular tools for the past decades. Full-length infectious clones are a good example of these tools as they allow the genetic manipulation (e.g. mutations, deletions, insertions) of viral genomes and further studies of the phenotypic effects of such modifications (Aubry et al. 2015).

Since the construction of the first flavivirus full-length clone (Rice et al. 1989), some technical difficulties have been reported, such as the instability of the viral genome in cloning bacterial hosts. To overcome part of the obstacles, several novel molecular techniques have been developed, including low-copy-number plasmids, bacterial artificial chromosomes and yeast-based homologous recombination. In addition, infectious clones or replicon systems expressing reporter genes (e.g. firefly and Renilla luciferases, green and yellow fluorescent proteins) have been developed and used mainly on molecular virology studies, to construct viral vectors and on sophisticated antiviral assays (De Baets et al. 2015, Patkar et al. 2009, Roth et al. 2009, Schoggins et al. 2012, van den Worm et al. 2012, Dag et al. 2013, Kato et al. 2014, Ding et al. 2015, Zhang et al. 2015). Among the 
reporter genes, Gaussia luciferase (GLuc) started to be used in in vivo and in vitro assays due to some advantages such as high stability and sensitivity, and the fact that it is secreted, facilitating its detection in several experiments (Tannous et al. 2005, Venisnik et al. 2007).

Considering the aforementioned, here we report the construction and characterization of a stable recombinant YFV (made by homologous recombination in yeast using YFV-17D) expressing the GLuc gene. We also show the use of YFV-GLuc in an antiviral assay as one of its several possible applications.

\section{MATERIALS AND METHODS}

\section{CELL AND VIRUS CULTURE}

Baby hamster kidney (BHK-21) cells were maintained at $37^{\circ} \mathrm{C} / 5 \% \mathrm{CO}_{2}$ in minimal essential medium (MEM) supplemented with 10\% fetal bovine serum (FBS), 1\% Penicillin (stock at $10.000 \mathrm{UI} / \mathrm{mL}$ ) and Streptomycin (stock at $10.000 \mu \mathrm{g} / \mathrm{mL}$ ) (LGC Biotecnologia, Cotia, SP, Brazil) and 0.1\% Fungizone (Gibco, Langley, OK, EUA). The YFV-17D virus, previously generated by Gil L.H.V.G. et al. (unpublished data) by pBSC-YFV17D transfection in BHK-21 cells, was amplified in BHK-21 cells, titrated by plaque assay and used as a positive control in experiments. The YFV-17D cloned genome was kindly provided by Galler, Fiocruz/RJ, Brazil.

CONSTRUCTION OF THE pBSC-YFV-GLuc PLASMID

pBSC-YFV-GLuc plasmid was constructed by homologous recombination in yeast on $\mathrm{pBSC}$-YFVYFP-DENV1linker, a recombinant virus with the YFP gene inserted in the YFV-17D genome (Fig. 1), previously made by Gil L.H.V.G. and AlmeidaQueiroz S.R.A (unpublished data) according to the strategy reported by Bonaldo et al. (2007). For this, pBSC-YFV-YFP-DENV1linker was digested with NarI and the GLuc PCR product, containing terminal sequences homologous to $\mathrm{E}$ and NS1 regions of the NarI-linearized pBSC-YFV-YFPDENV1linker, was directly cloned to produce the pBSC-YFV-GLuc recombinant plasmid. Digestion of pBSC-YFV-YFP-DENV1linker and the cloning strategy are shown in Fig. 1.

The GLuc reporter gene was amplified from the plasmid pGLuc-NS (WF10) (kindly provided by Perez, University of Maryland) through PCR with Taq Platinum (Invitrogen, Carlsbad, CA, EUA). PCR conditions were as follows: initial denaturation at $94^{\circ} \mathrm{C}$ for $30 \mathrm{~s}$; and 32 cycles of denaturation at $94^{\circ} \mathrm{C}$ for $15 \mathrm{~s}$, annealing at $52^{\circ} \mathrm{C}$ for $30 \mathrm{~s}$, and extension at $68^{\circ} \mathrm{C}$ for $1 \mathrm{~min}$. The recombination oligonucleotides (YFV-GLuc-F and YFV-GLuc-R) used to amplify the GLuc sequence are listed in Table I.

Briefly, pBSC-YFV-GLuc plasmid was generated by homologous recombination in Saccharomyces cerevisiae strain RFY206 and transformed by a procedure using lithium acetate (Sambrook and Russel 2001). The pBSC-YFVGLuc plasmid has a coding sequence for tryptophan (trp) as a marker for transformed cells selection on medium without trp. Then, selection of positive colonies was performed using Yeast Nitrogen Base without trp and plasmid DNA extraction was performed using QIAprep Miniprep Kit (Qiagen, Valencia, CA, USA). The DNA of the colonies was sequentially screened by two PCR reactions using Taq Platinum (Invitrogen) and the same PCR conditions mentioned above; oligonucleotide pairs were YFV-GLuc-F and YFV-NS1-2502-R, and YFV-2375-F and YFV-GLuc-R (Table I).

In vitro TRANSCRIPTION AND TRANSFECTION

For in vitro transcription, the full-length cDNA recombinant virus genome was amplified by PCR, using the KlenTaq-LA polymerase enzyme (Clontech, Mountain View, CA), and pBSC-RsrIIT7-5'-YFV-F and YFV-3'UTR-R oligonucleotides 
TABLE I

Oligonucleotides used for pBSC-YFV-GLuc construction and in vitro transcription.

\begin{tabular}{|c|c|}
\hline Oligonucleotide & Sequence \\
\hline YFV-Gluc-F ${ }^{a}$ & 'GACCAGGGCTGTGCAATTAATTTCGGGGGCGCCATGGGAGTCAAAGTTCTGTTTG \\
\hline YFV-Gluc-R ${ }^{\mathrm{b}}$ & 'CTCGGGCGGTTGCTTCGAACATTTTGGCGCCGTCACCACCGGCCCCCTTG \\
\hline $\begin{array}{l}\text { PBSC-RSRII-T7- } \\
\text { 5'-YFV-F }\end{array}$ & dCAAGCATGTAAATATCGTTTGAGTTCGGTCCGTAATACGACTCACTATAGAGTAAATCCTGTGTGCTAATTGAGG \\
\hline YFV-3'UTR-R & AGTGGTTTTGTGTTTGTCATCC \\
\hline YFV-NS1-2502-R & TCTCCGCACTTGAGCTCTC \\
\hline YFV - $2375-\mathrm{F}$ & ACAAGAAACATGACAATGTCC \\
\hline
\end{tabular}

${ }^{a}$ Forward oligonucletide. ${ }^{b}$ Reverse oligonucletide. ${ }^{c}$ Underlined nucleotide sequence corresponds to the region used for homologous recombination in yeast. ${ }^{\mathrm{d}}$ Bold nucleotide sequence corresponds to the $\mathrm{T} 7$ promoter for in vitro transcription.

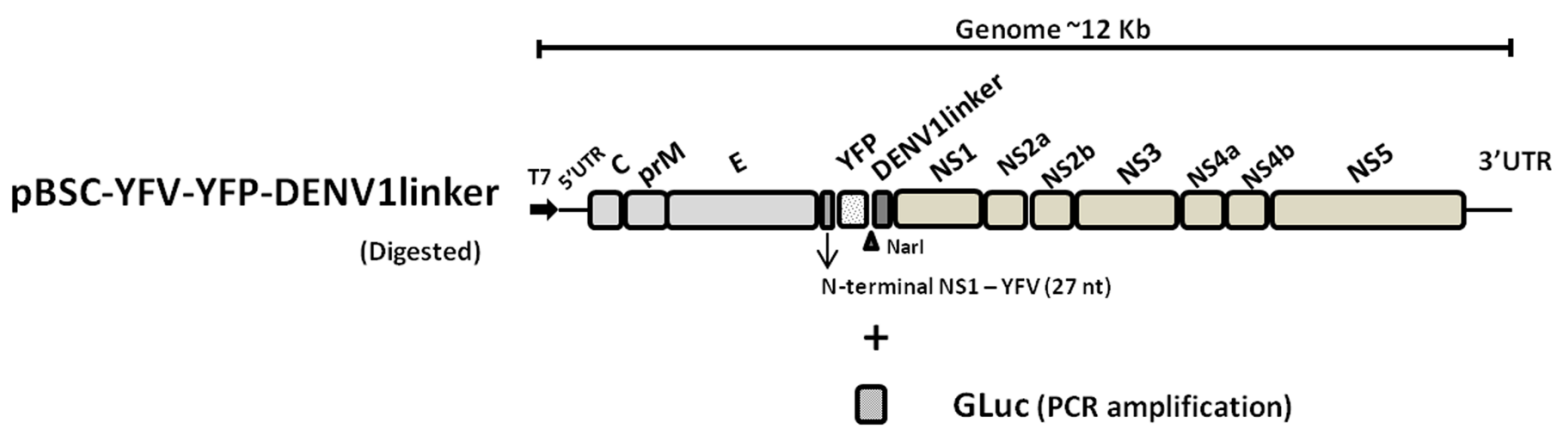

PBSC-YFV-GLUC

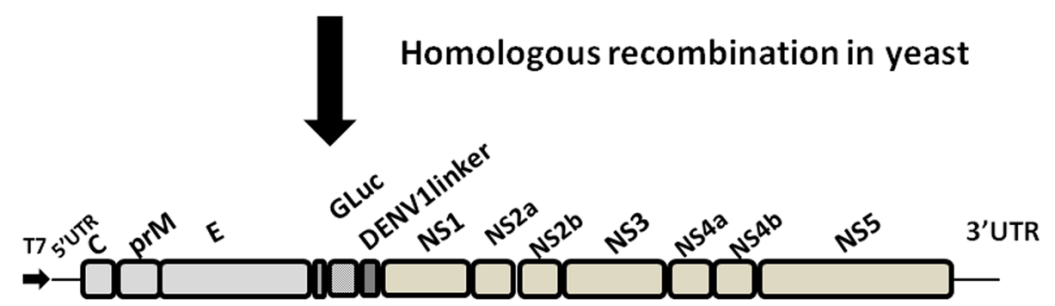

Figure 1 - Schematic representation of YFV-GLuc virus construction. pBSC-YFV-YFP-DENV1linker was digested with NarI restriction enzyme and the Gaussia luciferase (GLuc) reporter gene was amplified with recombination oligonucletides (YFVGLuc-F and YFV-GLuc-R) (Table I). The GLuc PCR product with homologous terminal sequences to NarI-linearized pBSCYFV-YFP-DENV1linker (E and NS1 region) was cloned to produce the pBSC-YFV-GLuc plasmid. DENV1linker: Stem-anchor E DENV1; UTR: untranslated region; NS: nonstructural; nt: nucleotide.

(Table I). The full-length PCR conditions were as follows: initial denaturation at $95^{\circ} \mathrm{C}$ for $5 \mathrm{~min} ; 32$ cycles of denaturation at $93^{\circ} \mathrm{C}$ for $1 \mathrm{~min}$, annealing at $56^{\circ} \mathrm{C}$ for $1 \mathrm{~min}$, and extension at $72^{\circ} \mathrm{C}$ for 13 min, with an increase of $10 \mathrm{~s}$ in extension per cycle; and a final extension at $72^{\circ} \mathrm{C}$ for $20 \mathrm{~min}$. The fulllength PCR product, which has the bacteriophage $\mathrm{T} 7$ promoter in the 5 ' terminal sequence, was purified by phenol-chloroform extraction followed by ethanol precipitation, and transcribed using 
the MEGAscript T7 kit (Ambion, Foster City, CA, USA) with the addition of the 7-methylguanosine cap analog for in vitro transcription (Ambion, Foster City, CA, USA). Full-length RNA transcripts were introduced into BHK-21 cells by electroporation as described previously (Santos et al. 2013). Transfected cells were plated in 6-well plates containing coverslips and virus generation was confirmed by immunofluorescence assay (IFA) and expression of the GLuc reporter gene, as described below. For these assays, coverslips or cell supernatants were collected at selected time points for further analyzes.

\section{INDIRECT IMMUNOFLUORESCENSE AND Gaussia LUCIFERASE ACTIVITY ASSAY}

Five days post-transfection, coverslips containing BHK-21 cells were collected and rinsed with phosphate buffered saline (PBS), fixed with cold acetone at $4^{\circ} \mathrm{C}$ for $5 \mathrm{~min}$ and air-dried. Cells were then incubated at $37^{\circ} \mathrm{C}$ for $1 \mathrm{~h}$ with a polyclonal hyperimmune mouse ascitic fluid raised against group B flaviviruses (kindly provided by Vasconcelos, Instituto Evandro Chagas) diluted 1:100 in PBS. After a washing step with PBS, cells were incubated with fluorescein isothiocyanate (FITC)-conjugated goat anti-mouse IgG antibody (Sigma-Aldrich, Saint Louis, MO, USA) diluted 1:100 in PBS at $37^{\circ} \mathrm{C}$ for $1 \mathrm{~h}$. Following a final washing step in PBS, cells were air-dried and the material was mounted on glass slides to allow visualization in a DMI 4000B fluorescence microscope (Leica, Wetzlar, Germany). At the same time point, the GLuc activity was measured in $10 \mu \mathrm{L}$ of cell supernatant using the Biolux Gaussia Luciferase Assay Kit (New England Biolabs, Ipswich, MA, USA) and Mithras LB 940 Multimode Microplate Reader (Berthold, Bad Wildbad, Germany).

\section{PHENOTYPIC CHARACTERIZATION}

YFV-GLuc (passage 4, p4) was used for phenotypic characterization. The recombinant virus was passaged four times in BHK-21 cells at a multiplicity of infection (MOI) of 1 (titrated by plaque assay) and incubated until $70-80 \%$ cytopathic effect was observed (CPE) (7-8 days).

\section{PLAQUE-FORMING ASSAY}

Plaque assay was performed to evaluate plaques formed by the parental virus (YFV-17D) and recombinant viruses. For this, monolayers of BHK21 cells were plated in a 6 -well tissue culture plate $\left(4 \times 10^{5}\right.$ cells $/$ well $)$ and $24 \mathrm{~h}$ later inoculated with serial 10 -fold dilutions $\left(10^{-1}\right.$ to $\left.10^{-5}\right)$ of YFV-17D or YFV-GLuc (p4). Following $1 \mathrm{~h}$ of adsorption at $37^{\circ} \mathrm{C}$ in $5 \% \mathrm{CO}_{2}$, the inoculum was removed. Cells were washed twice, overlaid with $1 \mathrm{~mL}$ of MEM containing 1\% agarose and 10\% FBS and incubated at $37^{\circ} \mathrm{C}$. After 7 days, plates were stained with Thiazolyl Blue Tetrazolium Bromide (MTT) in PBS and plaque morphology and size were analyzed. The staining procedure consisted of adding approximately $100 \mu \mathrm{L}$ of $5 \mathrm{mg} / \mathrm{mL}$ MTT in the wells and incubating plates for $2 \mathrm{~h}$ at $37^{\circ} \mathrm{C}$.

REPLICATION KINETICS OF YFV-GLuc VS. YFV-17D AND CURVE OF Gaussia LUCIFERASE EXPRESSION

The replication kinetics of YFV-GLuc and YFV$17 \mathrm{D}$ were analyzed in BHK-21 cells cultured in 24well plates $\left(8 \times 10^{4}\right.$ cells/well). Cells were infected with YFV-GLuc (p4) or YFV-17D virus at a MOI of 0.1 . The supernatant was collected for 4 days at every $24 \mathrm{~h}$ and frozen at $-80^{\circ} \mathrm{C}$. Viral RNA (vRNA) was extracted from $140 \mu \mathrm{L}$ of BHK-21 cell culture supernatant infected with YFV-GLuc or YFV17D with the Viral RNA Isolation Kit (MachereyNagel, Düren, Germany). vRNA quantification was performed by quantitative real-time PCR (qRTPCR) with the QuantiTect SYBR Green RT-PCR Kit (Qiagen, Valencia, CA, USA), using a standard 
curve of the PCR-targeted gene as described for dengue virus by Carvalho-Leandro et al. (2012). Primers used for YFV qRT-PCR were those used by Dash et al. (2012), targeting the 5' UTR and capsid gene junction of YFV. The positive control was RNA extracted from a known YFV-17D-infected culture. Reactions were performed in duplicate using the Applied Biosystems 7500 Fast Real-Time PCR System. The supernatants collected during the growth curve of YFV-GLuc were also used to perform the curve of GLuc expression. Finally, the relationship between GLuc activity and YFV-GLuc replication was evaluated by linear correlation and confirmed by $\mathrm{r}^{2}$ value. Both qRT-PCR and GLuc expression were performed twice (two separate biological assays).

\section{GENETIC STABILITY OF THE YFV-GLuc RECOVERED VIRUS}

To assess the genetic stability of the YFV-GLuc construct, the recombinant virus was successively passed in BHK-21 cells six times at MOI of 1 and incubated until 70-80\% CPE was observed (7-8 days). In passage 6 (p6), GLuc expression was evaluated three days post-infection. Moreover, vRNA was extracted from $140 \mu \mathrm{L}$ of supernatant from a BHK-21 cell culture infected with YFVGLuc using the Viral RNA Isolation Kit and the presence of GLuc gene was confirmed through RT-PCR using the One-Step RT-PCR Kit (Qiagen, Valencia, CA, USA), and YFV-2375-F and YFVNS1-2502-R oligonucleotides (Table I). The RTPCR conditions were: reverse transcription at $50^{\circ} \mathrm{C}$ for $30 \mathrm{~min}$; an initial PCR step (Taq activation) at $95^{\circ} \mathrm{C}$ for $15 \mathrm{~min}$; and 32 cycles of denaturation at $94^{\circ} \mathrm{C}$ for $1 \mathrm{~min}$, annealing at $52^{\circ} \mathrm{C}$ for $1 \mathrm{~min}$ and extension at $72^{\circ} \mathrm{C}$ for $1 \mathrm{~min}$. The integrity and identity of the RT-PCR products were evaluated by Sanger sequencing. Nucleotide analysis was performed with Ape-A plasmid Editor v1.10.4.

\section{ANTIVIRAL ASSAY}

In this assay, BHK-21 cells were cultured in 96well plates $\left(10^{4}\right.$ cells/well $)$ and $24 \mathrm{~h}$ later infected with YFV-GLuc (p4) and YFV-17D at a MOI of 0.1 . Immediately after adsorption, BHK-21 cells were treated with recombinant human alfa- $2 b$ interferon (IFN alfa-2b) (Heber Biotec, Havana, Cuba), a known antiviral molecule (Akira et al. 2001), at $1000 \mathrm{UI} / \mathrm{mL}$ by $48 \mathrm{~h}$. The negative controls were cells infected and not treated with IFN alfa-2b. After this step, GLuc activity was assessed according to the protocol described above and viral titer of YFV-17D was evaluated by plaque assay. This experiment was performed in duplicate.

\section{STATISTICAL ANALYSIS}

To compare the replication kinetics between the YFV-17D and YFV-GLuc viruses, Student's t-test (paired) was performed with the mean value of RNAv molecules $/ \mu \mathrm{L}(\log 10)$ at each timepoint (24, 48 and $72 \mathrm{~h})$ obtained in each experiment. A significance level of $5 \%$ was considered.

The correlation between GLuc activity and YFV-GLuc replication was evaluated by calculating the coefficient of determination $\left(r^{2}\right)$ after plotting in one graph data from both experiments.

\section{RESULTS}

\section{CONSTRUCTION OF pBSC-YFV-GLuc PLASMID}

The successful construction of pBSC-YFV-GLuc was confirmed by two PCR reactions using YFVGLuc-F and YFV-NS1-2502-R, and YFV-2375-F and YFV-GLuc-R oligonucletides (data not shown). The full-length cDNA recombinant virus genome was then amplified and in vitro transcribed, and the RNA was introduced into BHK-21 cells. The positive immunofluorescence and GLuc activity assay post-transfection (31-fold increase) showed that the viral protein and GLuc heterologous protein were correctly translated (Fig. 2). 
PHENOTYPIC CHARACTERIZATION OF YFV-GLuc IN CELL CULTURE

The plaque assay confirmed the efficient replication of the YFV-GLuc rescued virus in cell culture. However, at the $10^{-5}$ dilution YFV-GLuc plaques (p4) were smaller (5 $\mathrm{mm}$ mean diameter; eleven plaques were counted) than those formed by the YFV-17D parental virus $\left(10^{-4}\right)(9 \mathrm{~mm}$ mean diameter; seven were counted) (Fig. 3). The replication kinetics of the YFV-GLuc (p4) vs. YFV17D showed no statistical significance between the two viruses $(p<0.05)$ (Fig. 4a). The curve of GLuc activity on the supernatant of cells infected with YFV-GLuc (p4) showed a crescent GLuc expression over time (Fig. 4b). Moreover, a direct relationship between GLuc activity and YFV-GLuc replication was confirmed by regression analysis, with an $r^{2}$ value of 0.9967 (Fig. 4c).

a

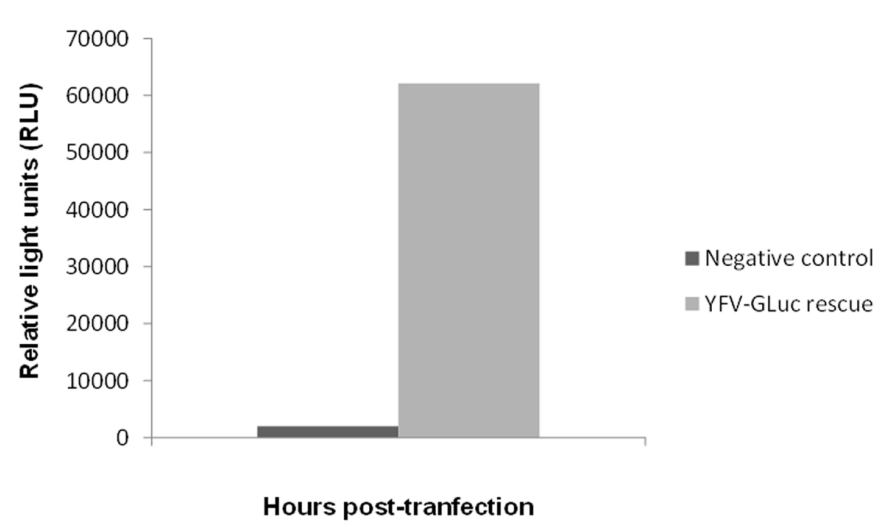

b
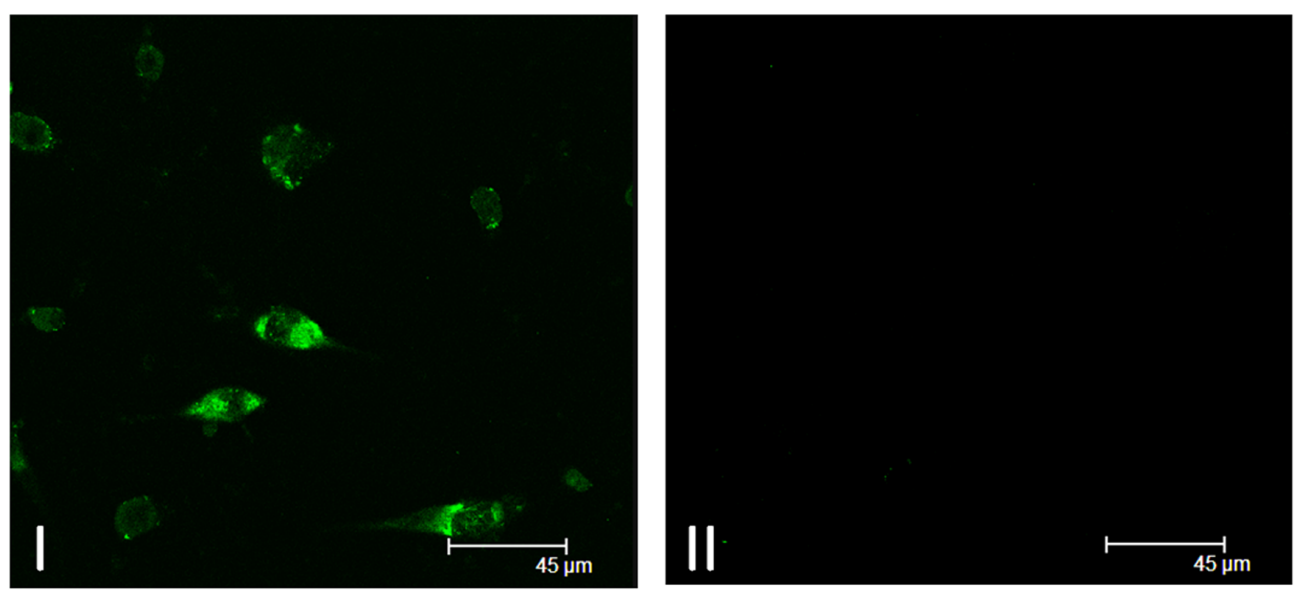

Figure 2 - a) Gaussia luciferase activity on the supernatant of BHK-21 cells 120 hours post-transfection with YFV-GLuc RNA. GLuc activity is expressed as relative light units (RLU). Negative control: supernatant of electroporated cells without YFV-GLuc RNA. b) IFA (63x magnification) performed with polyclonal hyperimmune mouse ascitic fluid raised against group B flaviviruses (primary antibody) and FITC-conjugated goat anti-mouse IgG antibody (secondary antibody). I) BHK-21 cells transfected with YFV-GLuc RNA at $120 \mathrm{~h}$ post-transfection; II) Negative control, mock-electroporated cells at 120 hours post-electroporation. 

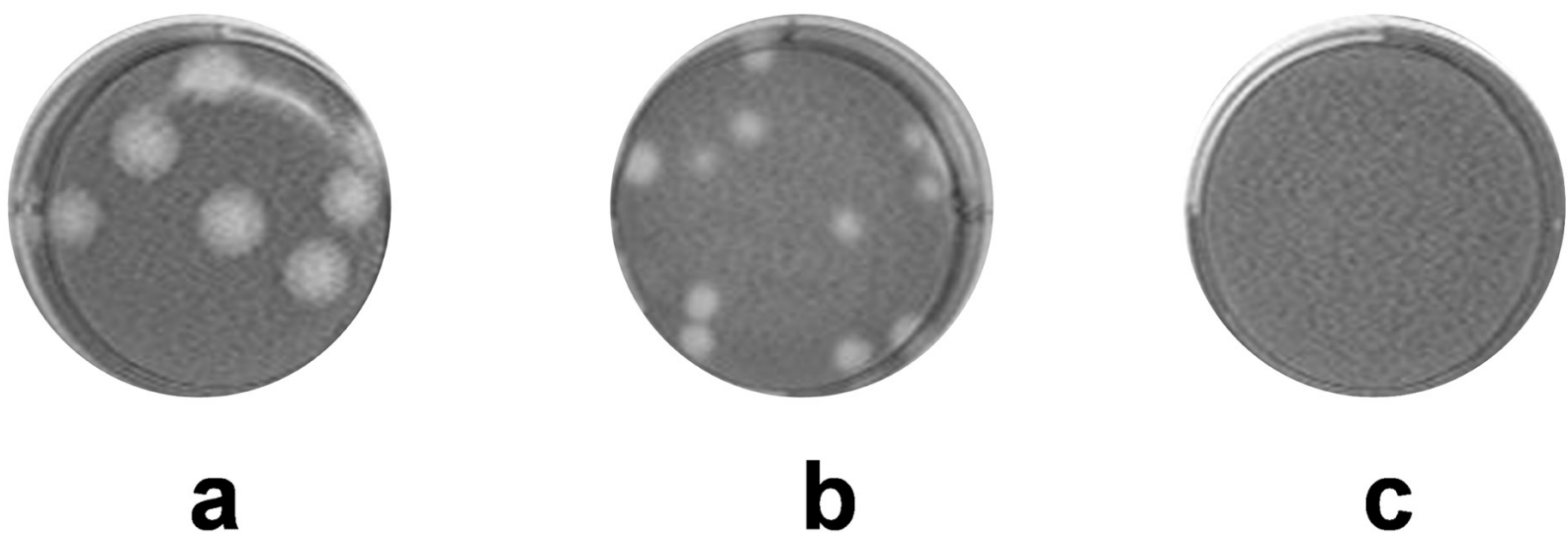

Figure 3 - Plaque assay of YFV-17D and YFV-GLuc. BHK-21 cells were inoculated with serial dilutions $\left(10^{-1}\right.$ to $\left.10^{-5}\right)$ of YFVGLuc or YFV-17D, and plaques were revealed 7 days post-infection. a) Plaques formed by YFV-17D at $10^{-5}$ dilution, measuring approximately $9 \mathrm{~mm}$ in diameter; b) Plaques formed by YFV-GLuc at $10^{-4}$ dilution, measuring approximately $5 \mathrm{~mm}$ in diameter; c) Negative control (non-infected cells).

\section{GENETIC STABILITY OF THE YFV-GLuc RECOVERED VIRUS}

The presence and integrity of the GLuc gene was confirmed after six passages in BHK-21 cells through RT-PCR (Fig. 5) and nucleotide sequencing (data not shown). Moreover, in p6, GLuc activity (130-fold increase) in relation to mock-infected cells confirmed the stability of GLuc expression.

\section{ANTIVIRAL ASSAY}

Conventional antiviral assays are performed through plaque assays (Green 2008) and are timeconsuming and require several steps. Thus, the YFV-GLuc recombinant virus was evaluated about its ability to be used as a novel antiviral assay platform: in this case, GLuc activity is inversely proportional to the antiviral activity of the evaluated substrate.

The antiviral assay was performed with IFN alfa-2b at $1000 \mathrm{UI} / \mathrm{mL}$. The result was expressed as the percentage of GLuc inhibition in BHK-21 cells infected by YFV-GLuc. Inhibition of GLuc expression in cells treated with IFN alfa-2b was $60 \%$ (similar inhibition results were observed with YFV-17D evaluated by plaque assay). The negative control consisted of cells infected and not treated with IFN alfa-2b.

\section{DISCUSSION}

Despite the success of the yellow fever vaccine with attenuated YFV (YFV-17D strain and its substrains YFV-17DD and YFV17D-204), yellow fever still poses a threat to several countries throughout the world. Although several drugs targeting the virus or blocking host responses are being developed, no anti-YFV drug is currently licensed (Julander 2013). Moreover, conventional assays to screen for antiviral compounds are usually performed through plaque assays and thus are laborious and time-consuming (Green et al. 2008). Repliconbased high-throughput screening methods using reporter genes represent important tools to evaluate antiviral compounds against several viruses in vitro and/or in vivo experiments (Patkar et al. 2009, Kato et al. 2014, Ding et al. 2015). However, replicon systems consist of a viral subgenome containing only nonstructural genes, and antiviral drugs or antibody targeting structural viral proteins cannot be evaluated through this technology (Mayhoub et al. 2011, Umamaheswari et al. 2011). To overcome 
Figure 4

a

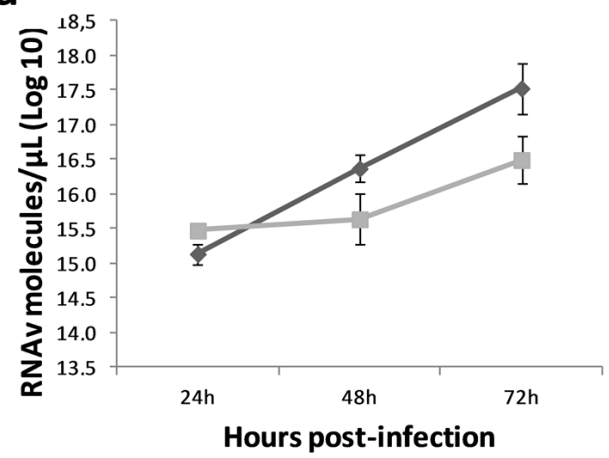

C

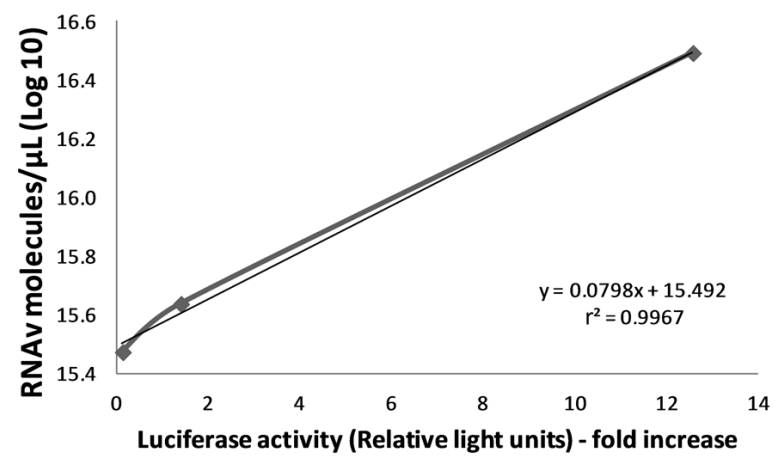

Figure 4 - a) Comparison of YFV-GLuc and YFV-17D replication kinetics. BHK-21 cells were inoculated with either virus at MOI of 0.1. At indicated times, the supernatant was collected and viral quantitation was assessed through SYBR Green-based quantitative real-time PCR. Each value represents the mean of two independent experiments; b) Curve of GLuc activity on the supernatant of cells infected with YFV-GLuc (MOI of 0.1). At different time points, the supernatant was collected and GLuc activity was measured and expressed as the fold-increase in relation to time zero using the relative light units. Each value represents the mean of two independent experiments. Each value represents the mean of two independent experiments; c) Linear correlation between GLuc activity (relative light units) and viral replication (RNAv molecules $/ \mu \mathrm{L}$ ). The replication kinetics of the YFV-GLuc and curve of Gaussia luciferase expression were overlapping and the direct relationship between them was evaluated by linear regression.

this limitation of replicon systems, strategies that use recombinant viruses containing reporter genes may be used.

Recombinant viruses expressing reporter genes (e.g. YFV, GFP, and firefly and Renilla luciferases) b
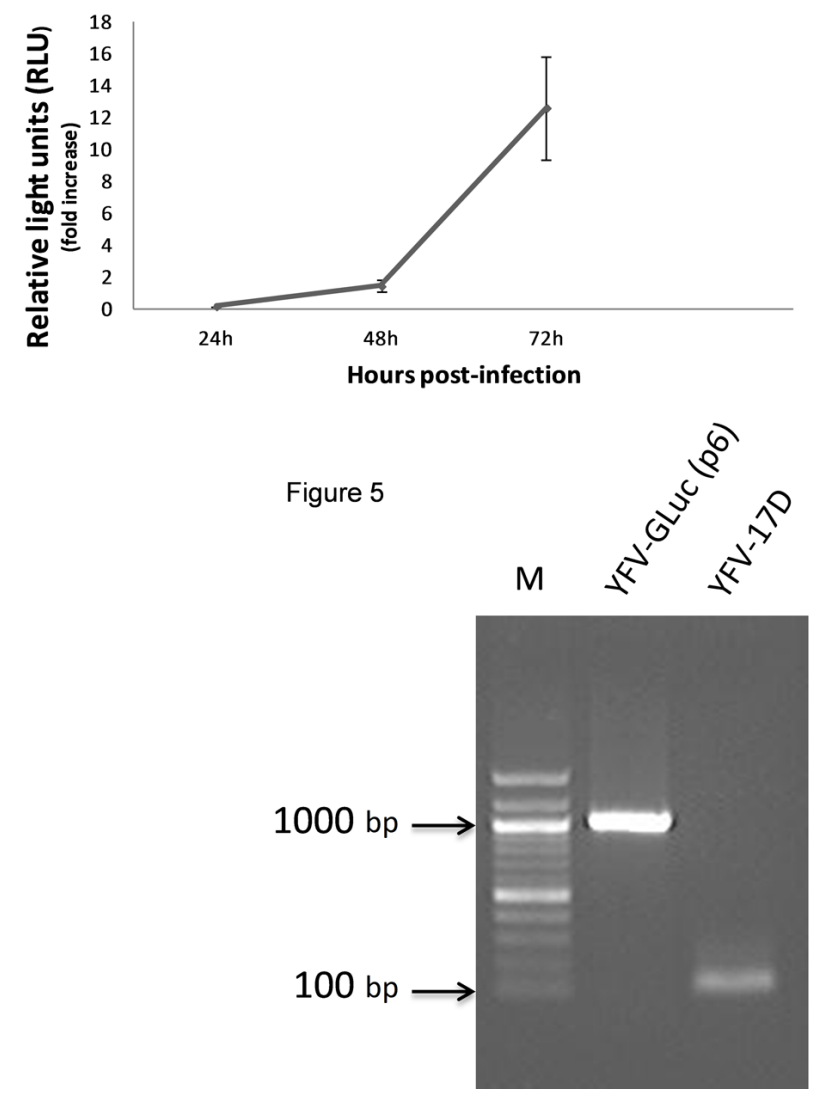

Figure 5 - RT-PCR of the YFV-GLuc (passage 6, p6). vRNA was extracted from BHK-21 cell culture supernatant infected with YFV-GLuc and YFV-17D parental virus and RT-PCR was performed to confirm the presence of the GLuc gene. The oligonucleotides set used in the amplification are listed in Table I (YFV-2375- F and YFV-NS1-2502-R). The difference between the size of YFV-GLuc (approximately 1000bp) and YFV-17D (approximately 130bp) amplicons is due to GLuc gene and accessory regions (Stem-anchor E DENV1 and NS1 N-terminal-YFV). M: 100bp DNA Ladder.

have been reported for distinct viruses and used for various purposes, e.g. on the evaluation of virus-host interactions, viral tropism, and antiviral and neutralization assays (Roth et al. 2009, Schoggins et al. 2012, van den Worm et al. 2012, Dag et al. 2013, De Baets et al. 2015, Zhang et al. 2015). These studies include the development of recombinant flavivirus expressing reporter genes, such as Dengue virus expressing GFP and firefly 
and Renilla luciferases (Zou et al. 2011, Schoggins et al. 2012). However, the instability or decreased fitness of the recombinant viruses compared to the parental virus limit their application in some experimental designs, as noted by Aubry et al. (2015). This instability in engineered virus expressing reporter genes was also reported in other flavivirus and DNA virus platforms (Song et al. 2012, Vandergaast et al. 2014, Zhang et al. 2015).

More recently, the constructions of viruses expressing GLuc as reporter gene and their use in several biotechnological applications have been reported. A desired feature of such clones is the stability of GLuc expression to allow their use in long-term studies, but unfortunately such condition has not always been achieved. Zhang et al. (2015) reported the construction of a West Nile Virus (WNV) expressing GLuc and its use in neutralization assays, but the recombinant virus gradually lost stability along the passages, where GLuc activity was no longer detected in passage 3. Vandergaast et al. (2014) also found similar instability in a GLuc-expressing WNV. The lower stability of these recombinant viruses in relation to YFV-GLuc probably is due to the insertion position GLuc gene (within C gene) and/or to the pathogenic strain used as backbone of WNV-GLuc, especially in Vandergaast et al. (2014). Conversely, in our experiments, GLuc gene was inserted into E-NS1 junction of an attenuated virus (YFV-17D). A recombinant poliovirus expressing GLuc retained the complete reporter gene for three passages and after that also gradually lost it (Song et al. 2012).

Our group has recently reported a recombinant bovine viral diarrhea virus stably expressing GLuc (Arenhart et al.2014) and now reports a recombinant YFV-17D stably expressing this same reporter gene (YFV-GLuc). The choice of GLuc as a reporter gene is mainly due to the following advantages: 1) high stability and sensitivity of GLuc (Tannous et al. 2005); 2) GLuc upon expression is secreted into the cell culture media or organics fluids/ secretions, thus cell lysis to assess GLuc activity is not necessary, making GLuc an ideal reporter gene for time-course studies (Venisnik et al. 2007); and 3) GLuc gene is smaller than other reporter genes, facilitating its manipulation in recombinant technologies.

In the present study, YFV-GLuc was stable after six passages in BHK-21 cells as indicated by RT-PCR (Fig. 5), sequencing integrity (data not shown) and GLuc activity (130-fold increase in comparison to mock-infected cells). The stability of GLuc activity also was evidenced by its increasing expression over time (Fig. 4b). The comparison of plaques formed by the parental and recombinant viruses in BHK-21 cells showed smaller-sized plaques in the latter (Fig. 3). Nevertheless, the replication kinetics of YFV-GLuc vs. YFV-17D showed no statistical significance between the two viruses $(p<0.05)$, although there was a tendency for YFV-GLuc to grow more slowly (Fig. 4a). The similarity between the YFV-17D parental virus and recombinant virus replication has already been reported in YFV platforms expressing heterologous proteins or small heterologous sequences from virus or protozoan at the E-NS1 expression site (Rumyantsev et al. 2010, Franco et al. 2010, Nogueira et al. 2013).

After construction, characterization and stability confirmation, YFV-GLuc was evaluated in an antiviral assay with IFN alfa-2b. Importantly, a direct relationship between GLuc activity and YFVGLuc replication was confirmed through regression analysis, indicating that GLuc expression may be used to represent viral replication (Fig. 4c). Thus, a decrease in GLuc activity after incubation with IFN alfa-2b reflects, indirectly, a decrease in viral genome replication. For instance, an inhibition of approximately $60 \%$ in GLuc activity after incubation with IFN alfa-2b reflects a decrease within the same range in viral replication. The result of the IFN-alfa $2 b$ assay shows the usefulness 
of GLuc-YFV construct to be used in further highthroughput antiviral screening assays.

In summary, the genetic manipulation of YFV-17D has proven to be stable in several biotechnological applications, as reviewed by Bonaldo et al. (2014). The present work reports the construction, characterization and additionally the use of a stable recombinant YFV-17D expressing GLuc to antiviral activity, combining in a single strategy the stability of the YFV-17D backbone and the advantages of GLuc as a reporter gene.

\section{ACKNOWLEDGMENTS}

This work has received financial support from the Conselho Nacional de Desenvolvimento Científico e Tecnológico (CNPq-470453/2012-5).

\section{REFERENCES}

AKIRA S, TAKEDA K AND KAISHO T. 2001. Toll-like receptors: critical proteins linking innate and acquired immunity. Nat Immunol 2: 675-680.

ARENHART S, FLORES EF, WEIBLEN R AND GIL LH. 2014. Insertion and stable expression of Gaussia luciferase gene by the genome of bovine viral diarrhea virus. Res Vet Sci 97: 439-448.

AUBRY F, NOUGAIREDE A, GOULD EA AND DE LAMBALLERIE X. 2015. Flavivirus reverse genetic systems, construction techniques and applications: a historical perspective. Antiviral Res 114: 67-85.

BONALDO MC, MELLO SM, TRINDADE GF, RANGEL AA, DUARTE AS, OLIVEIRA PJ, FREIRE MS, KUBELKA CF AND GALLER R. 2007. Construction and characterization of recombinant flaviviruses bearing insertions between E and NS1 genes. Virol J 4: 115.

BONALDO MC, SEQUEIRA PC AND GALLER R. 2014. The yellow fever 17D virus as a platform for new live attenuated vaccines. Hum Vacc Immunother 10: 12561265.

CARVALHO-LEANDRO D ET AL. 2012. Immune transcript variations among Aedes aegypti populations with distinct susceptibility to dengue virus serotype 2 . Acta Trop 124: 113-119.

DAG F, WEINGARTNER A, BUTUEVA M, CONTE I, HOLZKI J, MAY T, ADLER B, WIRTH D AND CICINSAIN L. 2013. A new reporter mouse cytomegalovirus reveals maintained immediate-early gene expression but poor virus replication in cycling liver sinusoidal endothelial cells. Virol J 10: 197.

DASH PK, BOUTONNIER A, PRINA E, SHARMA S AND REITER P. 2012. Development of a SYBR green I based RT-PCR assay for yellow fever virus: application in assessment of YFV infection in Aedes aegypti. Virol J 9: 27.

DE BAETS S, VERHELST J, VAN DEN HOECKE S, SMET A, SCHOTSAERT M, JOB ER, ROOSE K, SCHEPENS B, FIERS W AND SAELENS X. 2015. A GFP expressing influenza A virus to report in vivo tropism and protection by a matrix protein 2 ectodomain-specific monoclonal antibody. PLoS ONE 10: e01211491.

DING CB, ZHAO Y, ZHANG JP, PENG ZG, SONG DQ AND JIANG JD. 2015. A zebrafish model for subgenomic hepatitis $\mathrm{C}$ virus replication. Int J Mol Med 35: 791-797.

FRANCO D, LI W, QING F, STOYANOV CT, MORAN T, RICE CM AND HO DD. 2010. Evaluation of yellow fever virus $17 \mathrm{D}$ strain as a new vector for HIV-1 vaccine development. Vaccine 28: 5676-5685.

GREEN N, OTT RD, ISAACS RJ AND FANG H. 2008. Cell-based Assays to Identify Inhibitors of Viral Disease. Expert Opin Drug Discov 3: 671-676.

HANLEY KA, MONATH TP, WEAVER SC, ROSSI SL, RICHMAN RL AND VASILAKIS N. 2013. Fever versus fever: the role of host and vector susceptibility and interspecific competition in shaping the current and future distributions of the sylvatic cycles of dengue virus and yellow fever virus. Infect Genet Evol 19: 292-311.

ISHIKAWA T, YAMANAKA A AND KONISHI E. 2014. A review of successful flavivirus vaccines and the problems with those flaviviruses for which vaccines are not yet available. Vaccine 32: 1326-1337.

JULANDER JG. 2013. Experimental therapies for yellow fever. Antiviral Res 97: 169-179.

KATO F, KOBAYASHI T, TAJIMA S, TAKASAKI T, MIURA T, IGARASHI T AND HISHIKI T. 2014. Development of a novel Dengue-1 virus replicon system expressing secretory Gaussia luciferase for analysis of viral replication and discovery of antiviral drugs. Jpn J Infect Dis 67: 209-212.

LINDENBACH BD, MURRAY CL, THIEL H-J AND RICE CM. 2013. Flaviviridae: The Viruses and Their Replication. In: Knipe DM and Howley PM (Eds), Fields Virology, New York: Lippincott Williams \& Wilkins 6: 712-746.

MAYHOUB AS, KHALIQ M, KUHN RJ AND CUSHMAN M. 2011. Design, synthesis, and biological evaluation of thiazoles targeting flavivirus envelope proteins. J Med Chem 54: 1704-1714.

MONATH TP, SELIGMAN SJ, ROBERTSON JS, GUY B, HAYES EB, CONDIT RC, EXCLER JL, MAC LM, CARBERY B AND CHEN RT. 2015. Live virus vaccines based on a yellow fever vaccine backbone: standardized 
template with key considerations for a risk/benefit assessment. Vaccine 33: 62-72.

NOGUEIRA RT, NOGUEIRA AR, PEREIRA MC, RODRIGUES MM, NEVES PC, GALLER R AND BONALDO MC. 2013. Recombinant yellow fever viruses elicit CD8+ T cell responses and protective immunity against Trypanosoma cruzi. PLoS ONE 8: e59347.

PATKAR CG, LARSEN M, OWSTON M, SMITH JL AND KUHN RJ. 2009. Identification of inhibitors of yellow fever virus replication using a replicon-based highthroughput assay. Antimicrob Agents Chemother 53: 4103-4114.

RICE CM, GRAKOUI A, GALLER R AND CHAMBERS TJ. 1989. Transcription of infectious yellow fever RNA from full-length cDNA templates produced by in vitro ligation. New Biol 1: 285-296.

ROTH JP, LI JK, SMEE DF, MORREY JD AND BARNARD DL. 2009. A recombinant, infectious human parainfluenza virus type 3 expressing the enhanced green fluorescent protein for use in high-throughput antiviral assays. Antiviral Res 82: 12-21.

RUMYANTSEV AA, ZHANG ZX, GAO QS, MORETTI N, BROWN N, KLEANTHOUS H, DELAGRAVE S, GUIRAKHOO F, COLLETT MS AND PUGACHEV KV. 2010. Direct random insertion of an influenza virus immunologic determinant into the NS1 glycoprotein of a vaccine flavivirus. Virology 396: 329-338.

SAMBROOK J AND RUSSEL DW 2001. Molecular Cloning: A Laboratory Manual. n. 2, New York: Cold Spring Harbor Laboratory Press.

SANTOS JJ, CORDEIRO MT, BERTANI GR, MARQUES ET AND GIL LH. 2013. Construction and characterisation of a complete reverse genetics system of dengue virus type 3. Mem Inst Oswaldo Cruz 108: 983-991.

SCHOGGINS JW, DORNER M, FEULNER M, IMANAKA N, MURPHY MY, PLOSS A AND RICE CM. 2012.
Dengue reporter viruses reveal viral dynamics in interferon receptor-deficient mice and sensitivity to interferon effectors in vitro. Proc Natl Acad Sci USA 109: 1461014615.

SONG BH, YUN GN, KIM JK, YUN SI AND LEE YM. 2012. Biological and genetic properties of SA(1)(4)-14-2, a liveattenuated Japanese encephalitis vaccine that is currently available for humans. J Microbiol 50: 698-706.

TANNOUS BA, KIM DE, FERNANDEZ JL, WEISSLEDER R AND BREAKEFIELD XO. 2005. Codon-optimized Gaussia luciferase cDNA for mammalian gene expression in culture and in vivo. Mol Ther 11: 435-443.

UMAMAHESWARI A, KUMAR MM, PRADHAN D AND MARISETTY H. 2011. Docking studies towards exploring antiviral compounds against envelope protein of yellow fever virus. Interdiscip Sci 3: 64-77.

VAN DEN WORM SH ET AL. 2012. Reverse genetics of SARS-related coronavirus using vaccinia virus-based recombination. PLoS ONE 7: e32857.

VANDERGAAST R, HOOVER LI, ZHENG K AND FREDERICKSEN BL. 2014. Generation of West Nile virus infectious clones containing amino acid insertions between capsid and capsid anchor. Viruses 6: 1637-1653.

VENISNIK KM, OLAFSEN T, GAMBHIR SS AND WU AM. 2007. Fusion of Gaussia luciferase to an engineered anti-carcinoembryonic antigen (CEA) antibody for in vivo optical imaging. Mol Imaging Biol 9: 267-277.

ZHANG PT ET AL. 2015. Generation of a recombinant West Nile virus stably expressing the Gaussia luciferase for neutralization assay. Virus Res 211: 17-24.

ZOU G, XU HY, QING M, WANG QY AND SHI PY. 2011. Development and characterization of a stable luciferase dengue virus for high-throughput screening. Antiviral Res 91: 11-19. 